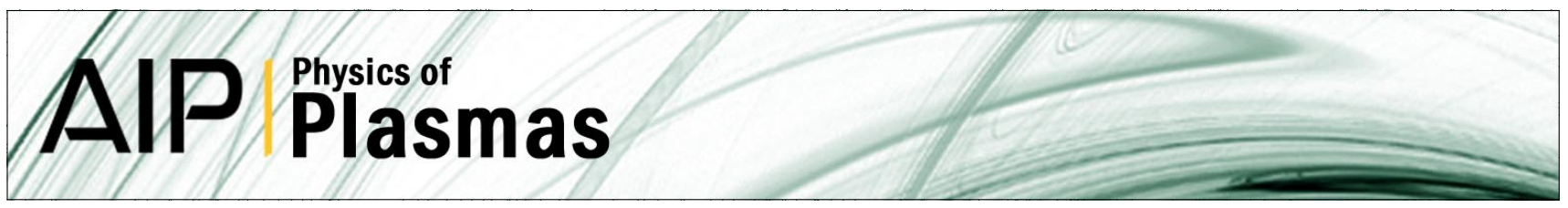

\title{
Particle-in-cell simulations of ambipolar and nonambipolar diffusion in magnetized plasmas
}

T. Lafleur and R. W. Boswell

Citation: Phys. Plasmas 19, 053505 (2012); doi: 10.1063/1.4719701

View online: http://dx.doi.org/10.1063/1.4719701

View Table of Contents: http://pop.aip.org/resource/1/PHPAEN/v19/i5

Published by the American Institute of Physics.

\section{Related Articles}

Kinetic Alfven double layer formed by electron viscosity

Phys. Plasmas 19, 072117 (2012)

Toroidal ripple transport of beam ions in the mega-ampère spherical tokamak

Phys. Plasmas 19, 072514 (2012)

Physics of the intermediate layer between a plasma and a collisionless sheath and mathematical meaning of the Bohm criterion

Phys. Plasmas 19, 073514 (2012)

Airflow influence on the discharge performance of dielectric barrier discharge plasma actuators

Phys. Plasmas 19, 073509 (2012)

A theoretical approach to electric breakdown behaviour and effective secondary emission coefficient in gas discharge process between two parallel-plane disk electrodes

J. Appl. Phys. 112, 023301 (2012)

\section{Additional information on Phys. Plasmas}

Journal Homepage: http://pop.aip.org/

Journal Information: http://pop.aip.org/about/about_the_journal

Top downloads: http://pop.aip.org/features/most_downloaded

Information for Authors: http://pop.aip.org/authors

\section{ADVERTISEMENT}

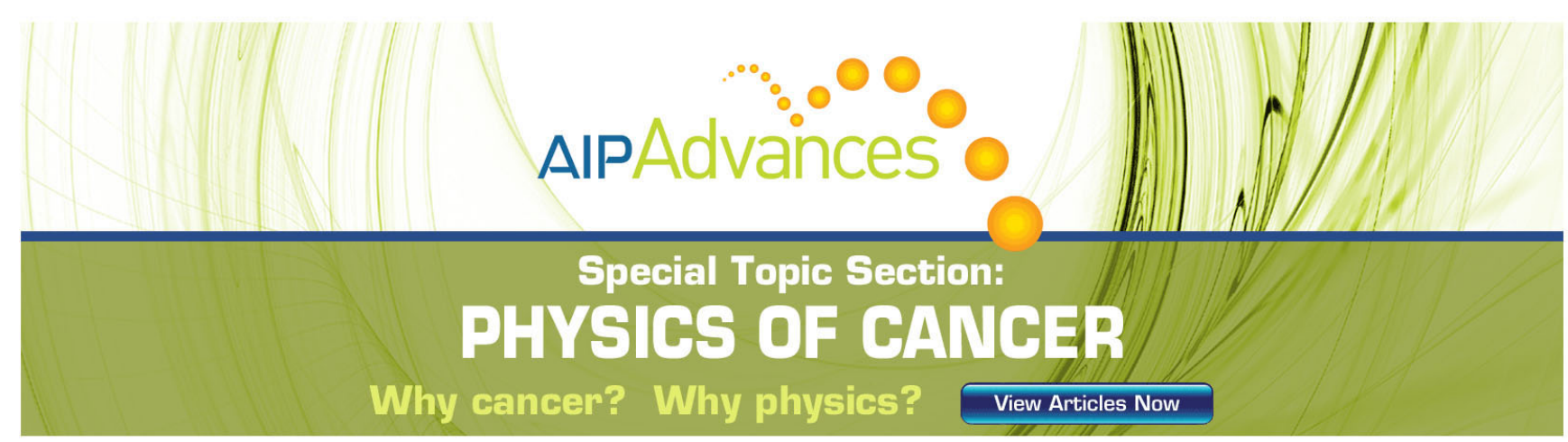




\title{
Particle-in-cell simulations of ambipolar and nonambipolar diffusion in magnetized plasmas
}

\author{
T. Lafleur ${ }^{\mathrm{a})}$ and R. W. Boswell \\ Space Plasma, Power and Propulsion Group, Research School of Physics and Engineering, The Australian \\ National University, Canberra ACT 0200, Australia
}

(Received 27 February 2012; accepted 10 April 2012; published online 23 May 2012)

Using a two-dimensional particle-in-cell simulation, we investigate cross-field diffusion in lowpressure magnetized plasmas both in the presence and absence of conducting axial boundaries. With no axial boundary, the cross-field diffusion is observed to be ambipolar, as expected. However, when axial boundaries are added, the diffusion becomes distinctly nonambipolar. Electrons are prevented from escaping to the transverse walls and are preferentially removed from the discharge along the magnetic field lines, thus allowing quasi-neutrality to be maintained via a short-circuit effect at the axial boundaries. (c) 2012 American Institute of Physics.

[http://dx.doi.org/10.1063/1.4719701]

\section{INTRODUCTION}

Plasma diffusion in the presence of magnetic fields is an important process in many areas of research, including fusion, ${ }^{1}$ space propulsion, ${ }^{2}$ and fundamental physics. ${ }^{1,3}$ In the absence of axial boundaries, cross-field diffusion (in the presence of an axially applied magnetic field) can be regarded as ambipolar, and since ions are typically only weakly magnetized, the diffusion behaviour is controlled by the more strongly magnetized electrons. ${ }^{1,3}$ This leads to a cross-field diffusion coefficient which is inversely proportional to the square of the magnetic field $\left(D_{\perp} \propto B^{-2}\right)$. A number of researchers have over the years reported anomalous diffusion behaviour, with cross-field diffusion coefficients scaling as $D_{\perp} \propto B^{-1,1,3}$ much larger than that predicted from classical diffusion theory. This behaviour is often explained by assuming the existence of electrostatic instabilities, which act to enhance the diffusion, as first postulated by Bohm. ${ }^{4,5}$

An alternative hypothesis put forward by $\operatorname{Simon}^{5,6}$ is that of the short-circuit effect. Simon's analysis questions the ambipolar assumption and demonstrates that the radial ion diffusion is free, not ambipolar. Electrons do not follow the ions across the magnetic field, but instead travel along field lines and complete the circuit at the axial boundaries. Thus, the electron current is short-circuited, and ambipolarity no longer requires equal ion and electron fluxes at each point on the boundary, but rather only that the total integrated fluxes over the entire surface be equal. The importance of short-circuit currents has been debated ${ }^{7}$ for many years; a debate that has recently been reignited ${ }^{8-14}$ by a number of theoretical ${ }^{15-17}$ and experimental ${ }^{18,19}$ works investigating plasma transport in magnetized discharges. The essential focus of these debates has been centered on the validity of the ambipolar assumption, and whether the short-circuit effect or Bohm-type diffusion offer viable explanations to the anomalous diffusion behaviour observed.

\footnotetext{
${ }^{a)}$ Electronic mail: trevor.lafleur@lpp.polytechnique.fr.
}

The difficulty involved in an accurate theoretical treatment of diffusion in two-dimensional (2D) magnetized plasmas is evident from the complex analytical formulations in such recent works as those in Refs. 15 and 16. One of the difficulties with most of these fluid models is the use of certain simplifying assumptions (such as ambipolarity) or boundary conditions used in order to obtain solutions (one recent exception is the work by Fruchtman ${ }^{20}$ ). In this paper, we approach the problem from a kinetic viewpoint and investigate diffusion behaviour by making use of $2 \mathrm{D}$ particle-in-cell (PIC) simulations. Because PIC simulations directly treat particle distribution functions, plasma production and transport can be self-consistently modelled, with no a priori assumptions required about whether ambipolarity does or does not occur. In the present work, we define the local ambipolarity condition as $\boldsymbol{\Gamma}_{e}=\boldsymbol{\Gamma}_{\mathbf{i}}$, where $\boldsymbol{\Gamma}_{e}$ and $\boldsymbol{\Gamma}_{i}$ are the electron and ion particle fluxes, respectively, while we define the global ambipolarity condition as $\oint_{A} \boldsymbol{\Gamma}_{e} \cdot d \mathbf{A}=\oint_{A} \boldsymbol{\Gamma}_{i} \cdot d \mathbf{A}$, where the integrals are performed over the surface area, $\mathbf{A}$, of the boundary walls. Diffusion behaviour is said to be ambipolar if the local ambipolarity condition is satisfied, while it is said to be nonambipolar if this condition is not met.

\section{SIMULATION MODEL}

We make use of a modified version of the custom developed PHOENIX 2D code previously described in Ref. 21. A schematic of the simulation domains used in the present investigation are shown in Fig. 1. We use a rectangular Cartesian geometry, with a uniform magnetic field applied in the $x$-direction (which we will refer to as the axial direction). The walls of the simulated system are all grounded, and thus the boundaries represent a conductor, which allows for the possible existence of currents flowing within the walls. We do not treat the case of insulating boundaries here, because of a number of potential subtleties concerning electronsurface collisions, as raised by $\operatorname{Simon}^{6,8}$ (see below). We set the axial system length to $L_{x}=10 \mathrm{~cm}$, while the transverse length is $L_{y}=5 \mathrm{~cm}$. Electrons and ions are moved with an explicit leap-frog scheme using the method described in 

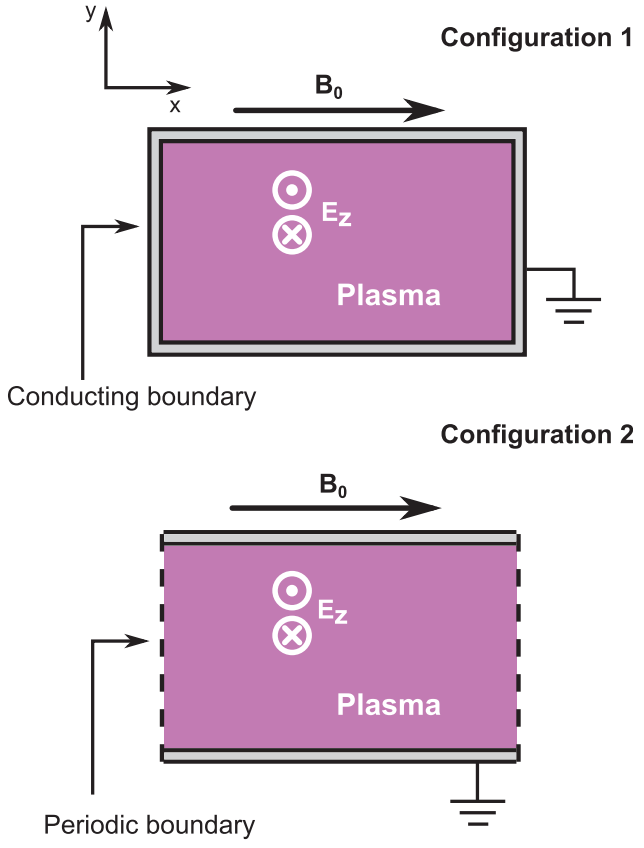

FIG. 1. Schematic of the PIC model indicating the two simulation configurations used. The applied magnetic field is spatially uniform, and the heating field, $E_{z}$, varies with time in the z-direction.

Ref. 22, while a standard Monte-Carlo collision (MCC) algorithm $^{23}$ is used to model electron-neutral (including elastic, excitation, and ionization reactions), and ion-neutral (including elastic and charge exchange reactions) collisions. The present study uses argon gas, which is considered to be uniformly distributed throughout the reactor. Particles that hit the boundaries are removed from the simulation, and secondary electron emission is turned off. Further code details and cross-section data used can be found in Ref. 21.

In order to model an inductive or helicon type reactor, we modify the electron heating scheme used by Meige $^{24}$ to two dimensions. Here, a time-varying electric field, $E_{z}$, is applied in the non-simulated $z$-direction, and whose magnitude varies depending on the electron current according to

$$
J_{0} \sin \omega t=J_{c}+\epsilon_{0} \frac{\partial E_{z}}{\partial t},
$$

where $J_{0}$ is a constant current density amplitude (the "antenna" current), $\omega$ is the angular excitation frequency $(13.56 \mathrm{MHz}), t$ is a time variable, $J_{c}$ is the electron particle current in the $z$-direction, and $\epsilon_{0}$ is the permittivity of free space. For a given $J_{0}$, Eq. (1) (which is numerically discretised) is solved for the heating field $E_{z}$. By including the particle current $J_{c}, E_{z}$ can vary in time and thus a feedback mechanism exists within the simulations. This allows a certain level of control over the maximum densities obtained and adds an additional element of reality to the heating mechanism. A number of different heating region models are used (representative of inductive or helicon heated discharges), as demonstrated in Fig. 2. For example, heating model $A$ in Fig. 2 is uniform in space and extends throughout the simulation domain. For this case, the particle conduction current, $J_{c}$, is given by
A

B
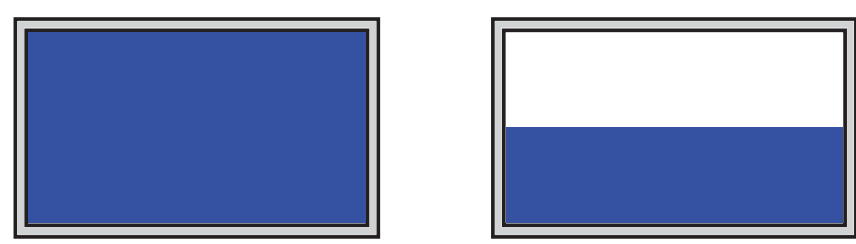

C

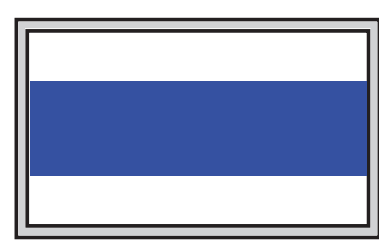

D

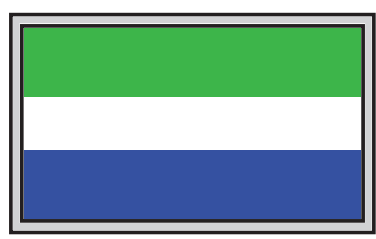

$$
\begin{aligned}
& E_{z}=E_{0} \sin \omega t \\
& E_{z}=-E_{0} \sin \omega t
\end{aligned}
$$

FIG. 2. Schematic of the different electron heating regions tested in the simulations. Each region has a spatially uniform electric field that varies with time.

$$
J_{c}=\frac{q q_{f}}{L_{x} L_{y}} \sum_{i} v_{z_{i}}
$$

Here, $q$ is the electron charge, $q_{f}$ is the macroparticle weight, $L_{x}$ and $L_{y}$ are the lengths of the axial and transverse sides of the rectangular simulation region, respectively, and $v_{z_{i}}$ is the $z$ velocity component of the $i$ th particle. By changing the magnitude of $J_{0}$, maximum plasma densities between $10^{15}$ $10^{16} \mathrm{~m}^{-3}$ are simulated, limited essentially by computational reasons because of the need to maintain numerical stability ${ }^{22}$ within the simulations. At these densities, the mean free path for Coulomb collisions is much larger than the system length, and so these types of collisions are neglected in the present simulations. Around $2 \times 10^{5}-5 \times 10^{5}$ electron-ion pairs (i.e., about $4 \times 10^{5}-1 \times 10^{6}$ total particles) are used, each representing about $2 \times 10^{7}$ real particles. The time steps used are around $5 \times 10^{-11} \mathrm{~s}$, sufficient to resolve both the electron plasma and electron cyclotron periods, while a few tens of thousands of grid points are used, sufficient to resolve the Debye length.

The simulation domains we study include an axially bounded discharge (configuration 1 in Fig. 1), and a domain with axially periodic boundary conditions (configuration 2 in Fig. 1). Configuration 1 models most realistic plasma reactors in which the axial length $L_{x}$ is of a similar order to the transverse length $L_{y}$, while configuration 2 models a system with a very long axial length, where short-circuit effects cannot occur, and thus the local ambipolarity condition, $\boldsymbol{\Gamma}_{e}=\boldsymbol{\Gamma}_{i}$, is expected to hold.

\section{RESULTS}

\section{A. General characterization}

We choose to focus on low-pressure plasmas in the presence of low to moderate magnetic fields, because of the 
importance of this regime in processing and propulsion reactors (see for example, the recent review paper by Charles, ${ }^{25}$ and reference therein). Although magnetic fields between 0-20 mTesla, and neutral pressures between 1-50 mTorr were simulated, in the results presented below, we choose to concentrate on discharges at 1 mTorr for four main test cases: (1) 0 mTesla bounded discharge (configuration 1 in Fig. 1), (2) 0 mTesla unbounded discharge (configuration 2 in Fig. 1), (3) 5 mTesla bounded discharge, and (4) 5 mTesla unbounded discharge. Since the unbounded cases should not show any axial variation of plasma properties, the axial simulation length, $L_{x}$, is shortened to reduce simulation times. For all of the test case results presented below, we make use of heating model $A$ in Fig. 2. Before running these test cases, the simulation code was checked to ensure that the theoretical particle cyclotron frequencies, and gyro-radii were correctly reproduced. The simulated pressures include electron-neutral mean-free paths in the range $0.5-20 \mathrm{~cm}$, the limits of which are smaller and larger than the simulation dimensions.

Time-averaged contour plots of the four test cases are shown in Fig. 3. The "antenna" current density, $J_{0}$, was chosen, so as to obtain a maximum plasma density of around $1-2 \times 10^{15} \mathrm{~m}^{-3}$ for each case. Cases (1)-(3) (Figs. 3(a), $3(\mathrm{c})$, and $3(\mathrm{~d})$ ) are all observed to reach equilibrium after about 20-30 $\mu$ s, which is approximately equal to the mean ion residence time. Case (4) (Fig. 3(b)) however is observed to take a much longer time to equilibrate and has not reached a steady state even after $200 \mu$ s. The maximum plasma potential for this case is only about $7 \mathrm{~V}$, which is much
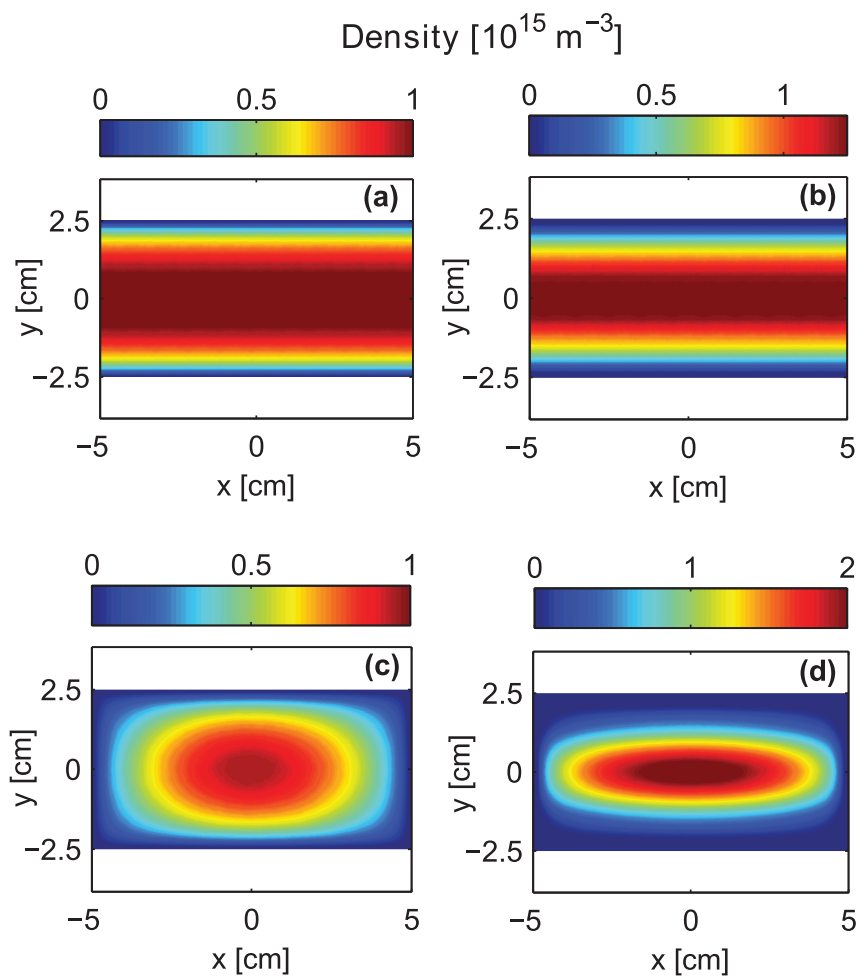

FIG. 3. Contour plots of the time-averaged electron density for (a) 0 mTesla periodic, (b) $5 \mathrm{mTesla}$ periodic, (c) 0 mTesla bounded, and (d) $5 \mathrm{mTesla}$ bounded discharges. To reduce simulation times, the unbounded periodic cases have shorter axial system lengths, but to aid clarity, the results have been repeated to generate the same discharge length as for the bounded cases. smaller than the maximum of about $20-30 \mathrm{~V}$ for cases (1)-(3). As the magnetic field is further increased for case (4), the simulations are observed to take increasingly longer times to equilibrate, and the maximum plasma potential continues to decrease, until for sufficiently large fields (depending on the pressure), the electric field is observed to change direction (i.e., it now points inwards away from the walls). This can be understood from classical diffusion theory; ${ }^{1,3}$ since the electrons are strongly magnetized, the ions are no longer the slowest cross-field diffusing species, and thus it follows that the plasma transport now proceeds on a time scale associated with the more strongly magnetized electrons. The above discussion serves as the first indication that enhanced diffusion behaviour is present for case (3), which is observed to equilibrate after a similar time compared to the 0 mTesla cases.

The unbounded cases in Fig. 3 show a density profile that varies only in the transverse direction, as expected from the periodic nature of the axial boundary conditions. The density is a maximum at the center of the discharge, and gradually decreases near the walls. Similar behaviour is observed for the bounded cases, except that plasma confinement (seen by the lower density near the walls) in the transverse direction is observed for case (3) (Fig. 3(d)). No significant confinement is seen in the axial direction, since the magnetic field is only applied in the axial direction.

\section{B. Ambipolar and nonambipolar diffusion}

The existence of ambipolar/nonambipolar diffusion can be shown explicitly by observing the time-averaged electron and ion particle fluxes to each of the simulation boundaries. This is shown in Fig. 4 for cases (1)-(4). Due to symmetry, we only show the fluxes to the top and right-hand walls for the bounded cases, and only the fluxes to the top wall for the unbounded cases. For both unbounded cases, the timeaveraged electron and ion current densities are spatially uniform, and approximately equal (slight fluctuations exist due to the statistical nature of the PIC simulations). This demonstrates that the local ambipolarity condition is satisfied, and thus that the flow is ambipolar, as expected due to the absence of axial walls.

For case (3), we see that the ion flux to the transverse walls is significantly higher than the electron flux, while on the axial walls, the electron flux is larger than the ion flux. The sum of electron and ion fluxes over all walls is however equal. This presents clear evidence that local ambipolarity is not occurring and highlights the fundamental point of this paper; ion diffusion to the transverse walls is nonambipolar, while electrons are preferentially lost to the axial walls, verifying the hypothesis of Simon ${ }^{5,6}$ for the conditions simulated here. These results are also qualitatively consistent with recent fluid simulations of an electron cyclotron resonance reactor ${ }^{26}$ (see for example Table II in this reference). The results for case (1) demonstrate that even in the absence of a magnetic field, the diffusion is also not completely ambipolar; because of the conducting boundaries, only global ambipolarity is required. This is consistent with the experimental and theoretical work in Ref. 27. In that paper, the authors took a series of 


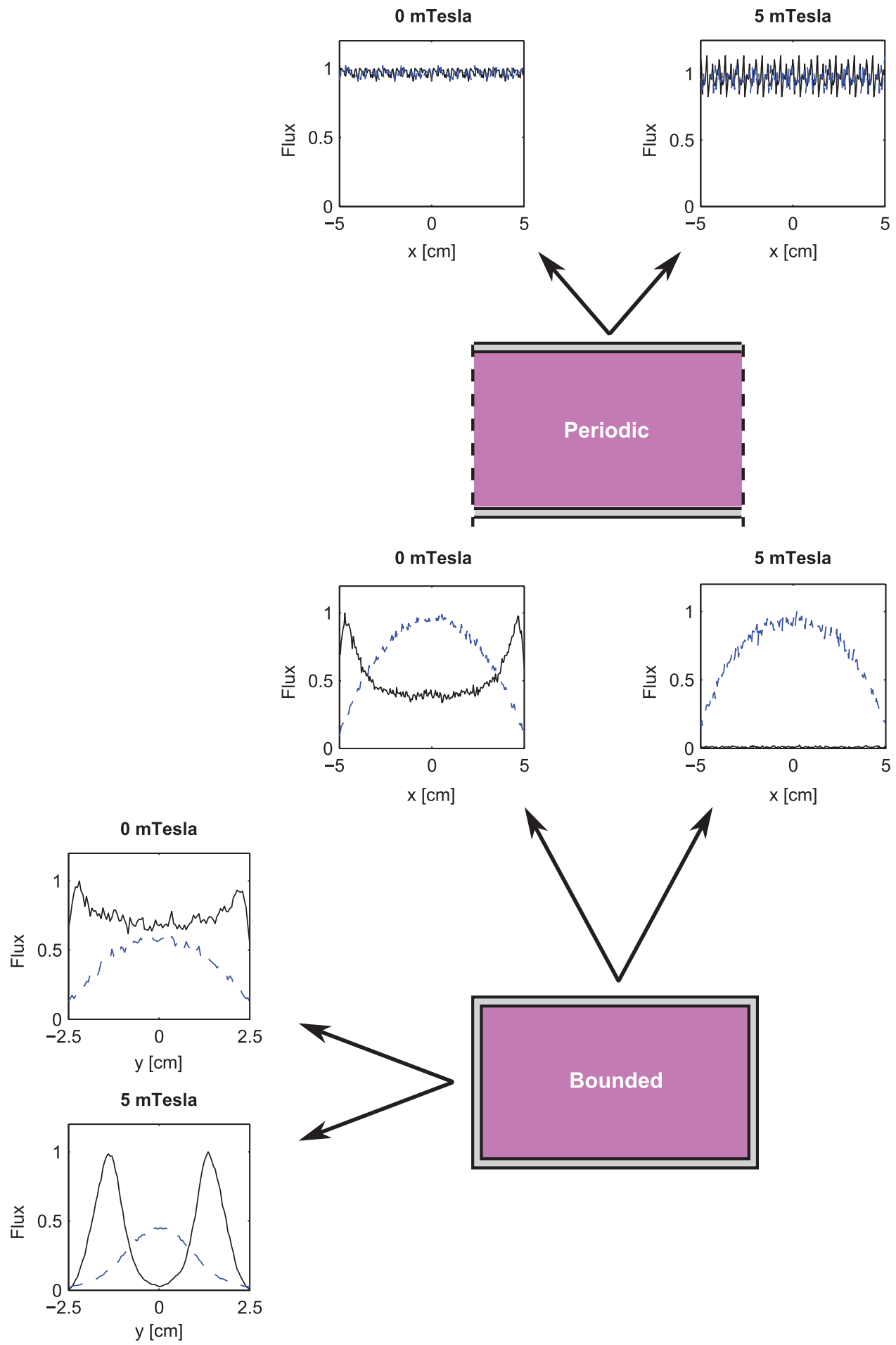

FIG. 4. Time-averaged particle fluxes along the transverse and axial walls for the periodic and bounded $0 \mathrm{mTesla}$ and 5 mTesla test cases. The dashed lines show the ion flux, while the solid lines show the electron flux. For the unbounded simulations, to aid clarity, the results have been repeated to generate the same discharge length as for the bounded cases. For the bounded simulations, the total sum of the axial and transverse electron and ion fluxes are equal.

measurements of the net current density on a conducting wall within an inductively coupled plasma and found a significant deviation from ambipolarity. They found a positive net current on the central region of the wall and a negative net current on the edges; behaviour consistent with a higher ion flux to the central region, and a higher electron flux at the edges (similar to the transverse flux profiles in Fig. 4). The authors also made use of a 2D kinetic model of the discharge and showed that the ion flux profile is peaked in the center and essentially follows the plasma density profile. Furthermore, the theoretical model predicted a slightly higher electron flux at the edges of the boundary, qualitatively similar to the simulation results obtained here. The higher flux at the edges was not as large as that of the simulation results however, but the system geometry is different, and the theoretical model made a number of assumptions (such as a spatially uniform electron energy distribution function). By setting $L_{x}=$ $L_{y}$ for the 0 mTesla simulation case, it is checked that the electron (ion) flux profiles on all walls are identical.

The ion flux loss profiles for the bounded cases are observed to be fairly similar for both the 0 mTesla and 5 mTesla cases and are peaked at the center of the walls. The axial electron flux loss profiles however have a local minima at the center and show a double-peaked profile. 
Since the plasma potential is largest in the center of the discharge (see below), the potential barrier seen by the electrons is greatest along the symmetry lines of the discharge, and thus it is perhaps not unexpected that the profiles should be peaked off-axis.

\section{Quasi-neutrality}

Figures 5(a) and 5(b) shows the transverse time-averaged density profiles for the bounded cases in the center of the discharge (i.e., at $x=0 \mathrm{~cm}$ ). The solid lines show the electron density, while the dashed lines show the ion density. The confining effect of the magnetic field is clearly seen by the narrower density profile for the 5 mTesla case (Fig. 5(b)) as compared to the 0 mTesla case (Fig. 5(a)). Also evident is the fact that quasi-neutrality is maintained within the bulk plasma, as seen by the similar ion and electron densities. This demonstrates that despite the negligible electron loss to the transverse walls for the $5 \mathrm{mTesla}$ case, electrons are able to remove all excess charge by travelling along the axial direction. For the same current density amplitude, $J_{0}$, the $5 \mathrm{mTesla}$ case has a slightly larger density in the center of the discharge, which can be understood from Eq. (2); because of confinement, the plasma is less dense near the walls and thus the density in the center can increase to maintain the same current amplitude.

\section{Plasma potential and Bohm velocity}

Figure 6(a) shows the time-averaged transverse plasma potential profiles for the bounded cases (at $x=0 \mathrm{~cm}$ ). As is seen, the profiles are symmetric and largest at the center of the discharge. The maximum plasma potential of about $30 \mathrm{~V}$
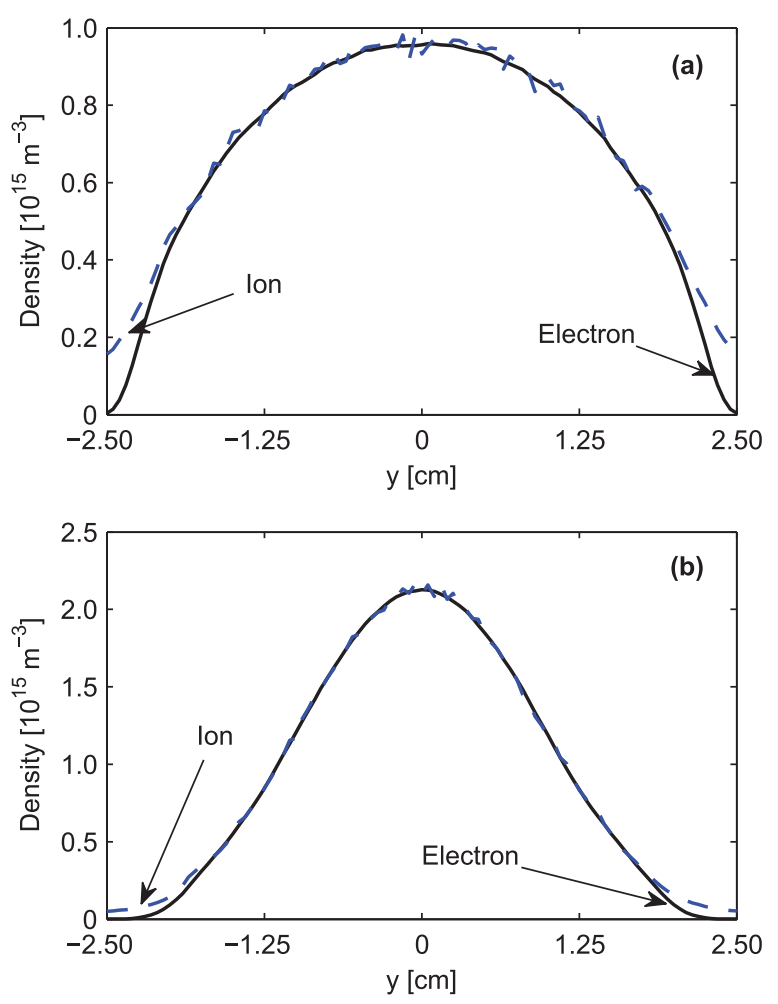

FIG. 5. (a) Time-averaged electron $\left(n_{e}\right)$ and ion $\left(n_{i}\right)$ densities in the transverse direction at $x=0 \mathrm{~cm}$ for the bounded (a) 0 mTesla and (b) 5 mTesla cases.
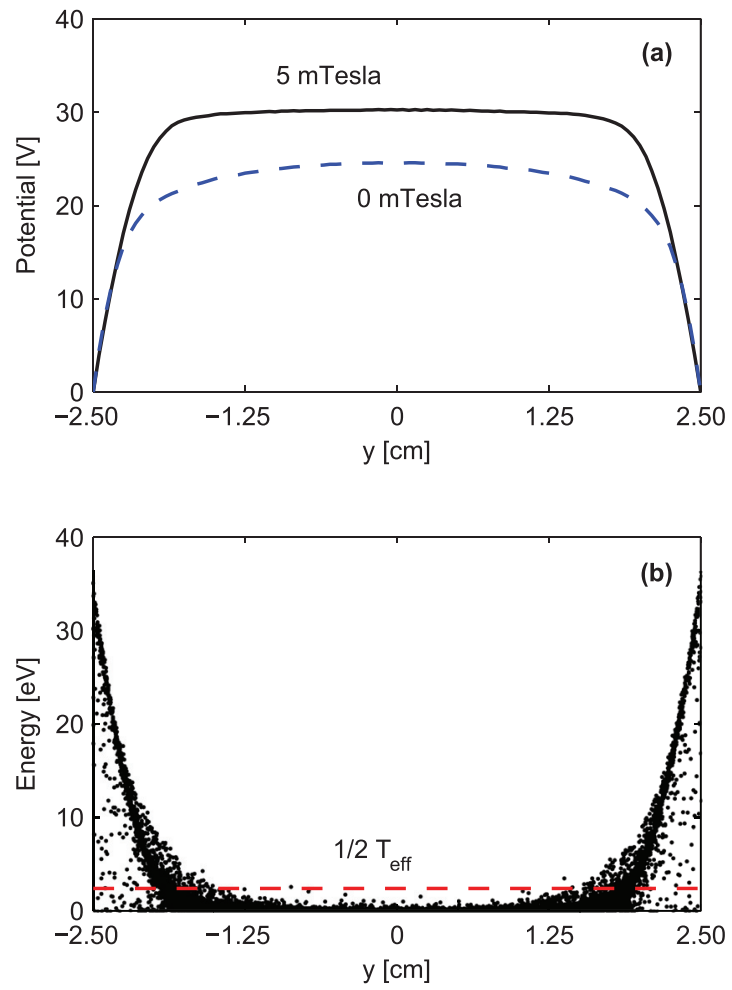

FIG. 6. (a) Time-averaged plasma potential in the transverse direction at $x=0 \mathrm{~cm}$ for the bounded $0 \mathrm{mTesla}$ and $5 \mathrm{mTesla}$ cases. (b) Ion energy phase space in the transverse direction for the $5 \mathrm{mTesla}$ case.

for the $5 \mathrm{mTesla}$ case is some $5-6 \mathrm{~V}$ higher than that for the $0 \mathrm{mTesla}$ case. The maximum plasma potential, $V_{w}$, at a grounded wall for a standard sheath (including the presheath potential drop) can be given by ${ }^{3}$

$$
V_{w}=\frac{T_{e}}{2}\left[1+\ln \left(\frac{M}{2 \pi m}\right)\right],
$$

where, $T_{e}$ is the electron temperature, and $m$ and $M$ are the electron and ion masses, respectively. By making use of an effective electron temperature (about $4-5 \mathrm{eV}$, found from $T_{\text {eff }}=2 / 3\langle E\rangle$, where $\langle E\rangle$ is the average electron energy $\left.{ }^{3}\right)$, Eq. (3) gives a maximum plasma potential of about 20-25 V. This is close to the maximum plasma potentials in Fig. 6(a). The ion energy phase space for the 5 mTesla case is shown in Fig. 6(b). The dashed line shows half the effective electron temperature in the simulation. The ions are observed to have an energy close to half this electron temperature near the sheath edges, and thus enter the sheath with a velocity of the order of the Bohm velocity, even though the majority of electrons leave the discharge along the field lines. The maximum ion energy at the walls is slightly larger than the maximum plasma potential due to slight rf oscillations in the plasma potential that are removed during the time-averaging in Fig. 6(a).

\section{E. Pressure effects}

For the other magnetic field conditions and pressures and also for the different electron heating regions in Fig. 2, similar behaviour to that presented above was seen; the majority of electrons flow out along the field lines, and the 

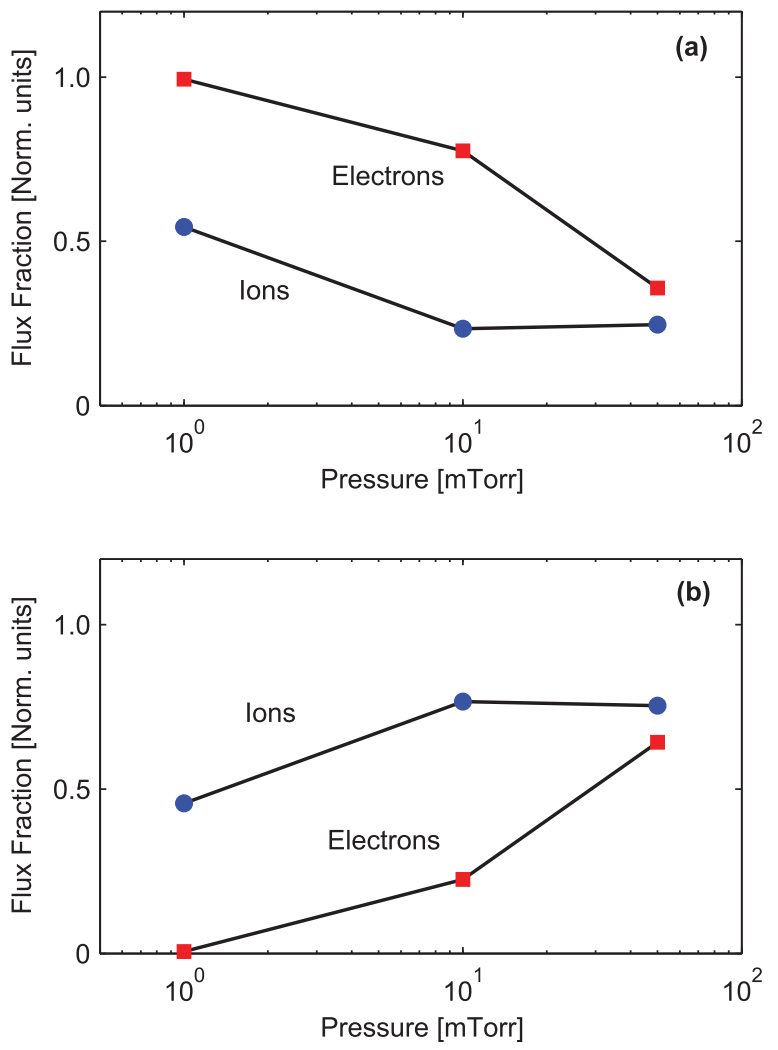

FIG. 7. Electron (closed squares) and ion (closed circles) particle flux fractions to the (a) axial and (b) transverse simulation walls as a function of pressure. The fluxes are normalized such that the sum of the axial and transverse components is equal to 1 .

diffusion is not ambipolar. Because of the electron heating field, $E_{z}$, together with the axial applied magnetic field, $B_{0}$, a time-varying (with a time-average of 0 ) $\mathbf{E} \times \mathbf{B}$ drift is present in the transverse direction, which could play a role in the electron transport. The use of the different heating models in Fig. 2 (and also the use of different axial heating region limits) was observed to give similar behaviour to that presented above however, although slight changes to the electron flux loss profiles were observed (for example, heating region $B$ in Fig. 2 shows only a single-peaked loss profile).

For a given magnetic field, as the pressure is increased, the electron flux to the transverse walls is observed to increase, while the flux to the axial walls decreases, and thus the electron flux loss fractions become similar to those for the ions. This is shown in Figs. 7(a) and 7(b) for a magnetic field of $B_{0}=5 \mathrm{mTesla}$ and for pressures between 1-50 mTorr, spanning regimes where the electron-neutral mean free path is, larger $(\sim 20 \mathrm{~cm})$, similar $(\sim 2-3 \mathrm{~cm})$, and smaller $(\sim 0.5 \mathrm{~cm})$ than the system dimensions. As the pressure increases, the electron-neutral mean free path decreases, and hence collisions become more frequent, reducing the confining effect of the applied magnetic field. The ion flux components also change with pressure, since the ion-neutral mean free path changes as well.

\section{DISCUSSION}

The results above demonstrate the importance of axial boundaries in magnetized discharges and show that in general, the diffusion need not be ambipolar; short-circuit currents can play a significant role and change the overall transport behaviour. In systems with much longer axial lengths, we might imagine that these short-circuit currents begin to play less important roles, but since the parallel electron conductivity is orders of magnitude greater than the perpendicular conductivity, this required length could be quite large (depending on the neutral pressure). Nevertheless, since we have only simulated systems where $L_{x} \sim L_{y}$ (due to computational reasons), we cannot question recent experimental results ${ }^{18}$ that have claimed to observe Bohm type diffusion (a claim challenged by Simon. ${ }^{12,14}$ ).

A natural question to ask is whether the local ambipolarity condition can be recovered if the boundaries are insulating instead of conducting. Experimental results at the Oak Ridge National Laboratory ${ }^{28}$ have suggested that insulating boundaries do little to change the diffusion behaviour, and Simon $^{5,6}$ has suggested that the existence of electron-surface collisions could allow electrons to travel radially at the boundaries, so that a short-circuit effect can still be produced. In many fluid and PIC simulations, insulators are treated as surfaces or objects within the simulation domain with a known dielectric constant and onto which charge can be deposited by plasma currents. With this treatment, electronsurface collisions are not modelled, and thus if Simon is to be believed, the correct cross-field diffusion behaviour would not be able to be reproduced. Future work will require a far more careful approach to the treatment of insulators before the validity of the results presented here can be extended to insulating boundaries.

\section{CONCLUSION}

In summary, by making use of a self-consistent 2D PIC simulation, we have shown that for discharges bounded by conducting walls, the cross-field diffusion need not be ambipolar; electrons escape along the field lines and maintain quasi-neutrality via a short-circuit effect at the axial boundaries. Global ambipolarity is of course still maintained, but this condition only requires that the integrated electron and ion fluxes over the boundary surfaces be equal, not the individual fluxes at each location. While there is some experimental evidence in the literature to suggest that the use of insulating instead of conducting boundaries has little effect in restoring ambipolarity, we cannot yet conclude from the simulations that this is true.

\section{ACKNOWLEDGMENTS}

The authors would like to thank Professor A. Fruchtman for a number of useful discussions.

${ }^{1}$ F. F. Chen, Introduction to Plasma Physics and Controlled Fusion, Plasma Physics, 2nd ed. (Springer, New York, 2006), Vol. 1.

${ }^{2}$ D. M. Goebel and I. Katz, Fundamentals of Electric Propulsion: Ion and Hall Thrusters (Wiley, New Jersey, 2008).

${ }^{3}$ M. A. Lieberman and A. J. Lichtenberg, Principles of Plasma Discharges and Materials Processing, 2nd ed. (Wiley, New Jersey, 2005).

${ }^{4}$ A. Guthrie and R. K. Wakerling, The Characteristics of Electrical Discharges in Magnetic Fields (McGraw-Hill Book Company, New York, 1949). 
${ }^{5}$ A. Simon, Phys. Rev. 98, 317 (1955).

${ }^{6}$ A. Simon, in International Conference on the Peaceful Uses of Nuclear Energy, 2nd ed. (United Nations, Geneva, Switzerland, 1958), Vol. 33, p. 343.

${ }^{7}$ K. H. Geissler, Plasma Phys. 10, 127 (1968).

${ }^{8}$ A. Simon, Phys. Plasmas 14, 084703 (2007).

${ }^{9}$ V. Godyak, N. Sternberg, and D. Hoffman, Phys. Plasmas 14, 084704 (2007).

${ }^{10}$ A. Simon, Plasma Sources Sci. Technol. 17, 028001 (2008).

${ }^{11}$ A. Fruchtman, G. Makrinich, and J. Ashkenazy, Plasma Sources Sci. Technol. 17, 028002 (2008).

${ }^{12}$ A. Simon, Phys. Plasmas 15, 022507 (2008).

${ }^{13}$ J. E. Maggs and T. A. Carter, Phys. Plasmas 15, 074701 (2008).

${ }^{14}$ A. Simon, Phys. Plasmas 15, 074702 (2008).

${ }^{15}$ A. Fruchtman, G. Makrinich, and J. Ashkenazy, Plasma Sources Sci. Technol. 14, 152 (2005).

${ }^{16}$ N. Sternberg, V. Godyak, and D. Hoffman, Phys. Plasmas 13, 063511 (2006).
${ }^{17}$ D. Curreli and F. F. Chen, Phys. Plasmas 18, 113501 (2011).

${ }^{18}$ J. E. Maggs, T. A. Carter, and R. J. Taylor, Phys. Plasmas 14, 052507 (2007).

${ }^{19}$ S. D. Baalrud, N. Hershkowitz, and B. Longmeir, Phys. Plasmas 14, 042109 (2007).

${ }^{20}$ A. Fruchtman, Plasma Sources Sci. Technol. 18, 025033 (2009).

${ }^{21}$ T. Lafleur and R. W. Boswell, Phys. Plasmas 19, 023508 (2012).

${ }^{22}$ C. K. Birdsall and A. B. Langdon, Plasma Physics Via Computer Simulation (McGraw-Hill, New York, 1985).

${ }^{23}$ V. Vahedi and M. Surendra, Comput. Phys. Commun. 87, 179 (1995).

${ }^{24}$ A. Meige, R. W. Boswell, C. Charles, and M. M. Turner, Phys. Plasmas 12, 052317 (2005).

${ }^{25}$ C. Charles, J. Phys. D: Appl. Phys. 42, 163001 (2009).

${ }^{26}$ G. J. M. Hagelaar, Plasma Sources Sci. Technol. 16, S57 (2006).

${ }^{27}$ G. Mümken and U. Kortshagen, J. Appl. Phys. 80, 6639 (1996).

${ }^{28}$ A. Simon and R. V. Neidigh, Oak Ridge National Laboratory, Oak Ridge, TN, Report No. ORNL-1890, 1955. 